\title{
THE SEPARATION OF OVERLAPPING PEAKS IN CYCLIC VOLTAMMETRY BY MEANS OF SEMI-DIFFERENTIAL TRANSFORMATION
}

\author{
Marcin Palys, * Tomas Korba, Martinus Bos and Willem E. van der Linden \\ Laboratory of Chemical Analysis, Department of Chemical Technology, Twente University of Technology, \\ P.O. Box 217, 7500AE Enschede, The Netherlands
}

(Received 1 October 1990. Accepted 22 January 1991)

\begin{abstract}
Summary-A method for extracting single peaks from complex linear sweep and cyclic voltamperograms is presented. Voltamperograms are transformed by means of semidifferentiation, then all undesired peaks are removed from the semiderivative curve and replaced by calculated baselines. The resulting curve is semiintegrated back, giving a voltamperogram with one peak only. Baselines in the semiderivative domain are determined by the least-squares curve-fitting of datapoints from peak border regions, using the equation that describes the semiderivative peak of a reversible electrode process. With this procedure peaks can be removed without assumptions about the mechanism of the underlying electrode reaction. Due to its design, the algorithm presented is suitable for the fully automatic processing of cyclic and linear sweep voltamperograms. Performance of the procedure was checked with generated reversible voltamperograms as well as in real experiments with both reversible and irreversible systems. The smallest distance between two peaks of equal height, for which the described method can yield correct results, has been found to be $110 \mathrm{mV}$ for a reversible one-electron process at $298 \mathrm{~K}$. This procedure can also be applied to the elimination of the cathodic current from the cyclic voltamperogram of a single component in order to get a pure anodic current value, free from cathodic contribution, or vice versa.
\end{abstract}

Linear sweep voltammetry (LSV) and cyclic voltammetry $(\mathrm{CV})$ are simple and relatively fast electroanalytical techniques used mainly for qualitative studies. Their experimental simplicity and well-established theoretical background also make them attractive for automated electrochemical investigations to be implemented in an expert system for the elucidation of electrode mechanisms, and this is under study. However, applicability of LSV and CV to quantitative studies is restricted by the shape of the signal: a broad, asymmetric, "tailing" peak. As a consequence of this feature, in composite voltamperograms peaks often overlap and measurement of their parameters (peak current and peak potential) is significantly hindered. Influence of the cathodic current on the anodic part of the voltamperogram makes measurement of the anodic peak current difficult, or even impossible if several cathodic peaks exist. Therefore, a good method for the separation of overlapping peaks is desired, if one wants to use these techniques to obtain quantitative results. For

*On leave from University of Warsaw, Chemistry Department. Author for correspondence. our purposes, we would demand the method to have additional features: the ability to separate peaks without any special assumptions about the electrode reaction mechanism and the capability to resolve peaks originating from a multistep reaction of one compound as well as from signals obtained from the mixture of the electroactive species.

The separation of cyclic voltammetric peaks has been an objective of extensive studies. ${ }^{1-4}$ However, most popular methods have some restrictions: direct curve fitting of overlapping peaks ${ }^{1}$ requires parameters describing the peak shapes before fitting can start, knowledge of what excludes its application to unknown (or unidentified) mixtures and to peaks resulting from multistep reactions. The deviation-pattern recognition method ${ }^{2}$ needs knowledge about the underlying electrode reaction mechanism. The approach of Perone et $a l^{3}{ }^{3}$ works only under supervision of the experimenter, relying on his decision. Interrupt-scan method, as proposed by Boudreau and Perone, ${ }^{4}$ while profitable for qualitative measurements, according to the authors doesn't give satisfactory quantitative results for staircase voltammetry. An extension of work ${ }^{1}$ with another, more general approxi- 
mate function ${ }^{4}$ to fit overlapping peaks retains the restrictions of the original work, i.e., the necessity of delivering peak-shape parameters prior to the fitting; moreover, the paper ${ }^{4}$ presents results of this method for square-wave voltammetry only.

Problems with overlapping peaks can be partially overcome by combining LSV with a mathematical transformation like semidifferentiation or semiintegration. ${ }^{5,6}$ The transformed voltamperograms reveal attractive features for both analytical and mechanistic studies, such as the independence of the signal on the potential change function, ${ }^{7}$ higher symmetry and small peak widths, ${ }^{5,8}$ a linear relationship between semiintegrated current and the surface concentration of the reactant at the electrode $e^{-11}$ and simpler analytical equations describing the peak shapes.

The increasing number of applications of semidifferentiation and semiintegration coupled to linear sweep voltammetry (also called semidifferential/semiintegral electroanalysis or convolution potential sweep voltammetry) is reflected by the fact, that recently commercially available instrumentation is equipped with these possibilities (e.g., Bioanalytical Systems Inc, PARC EG \& G Corp.). However, the number of electrode processes, for which a full theoretical description of the signals is available is rather small. As far as semiintegration is concerned, Saveant and co-workers published a series of papers, ${ }^{12-17}$ in which a number of equations has been derived. These equations, employing both current and its semiintegral cannot be used, however, for the direct interpretation of semiintegrated curves. For semidifferentiated voltamperograms, Goto and Ishii ${ }^{5}$ reported an equation describing the peak of the reversible process, while Dalrymple-Alford et $a^{6,{ }^{6,18}}$ gave the result for the irreversible case. Goto and Ishii ${ }^{8}$ also investigated the influence of the use of staircase instead of linear sweep voltammetry.

In contrast to semiderivative voltammetry, the theory of cyclic voltammetry is well developed. For mechanistic investigations, voltammetric curves can be studied according to the standard procedures if the necessary parameters are available. These procedures, most employing variations of peak potential, $E_{\mathrm{p}}$, peak current, $i_{\mathrm{p}}$, and anodic to cathodic current ratio with scan rate and concentration, often require values of currents which cannot always be directly measured from the recorded curve.
In this paper an approach is proposed, which combines the benefits of the easy interpretation of voltamperograms and the enhanced resolution characteristics of semidifferentiated linear sweep and cyclic voltammetry curves. This goal is achieved by semidifferentiation of a complex voltamperogram, separation of the semiderivative signals, removal of the undesired peaks by replacing them with the appropriate fragments of the baseline and semiintegration back to the $i(E)$ form, that will now contain peaks of interest only. Moreover, the proposed method is relatively universal and can be applied to electrochemical processes with different kinetics.

\section{THEORY}

The current in a linear sweep voltamperogram with two overlapping peaks can be described as the sum of two independent current functions, $i(t)=i_{1}(t)+i_{2}(t)$. Due to the linear and homogeneous character of the semidifferential operator, ${ }^{9,19}$ this additivity remains valid also in the semiderivative domain:

$$
\frac{\mathrm{d}^{1 / 2}}{\mathrm{~d} t^{1 / 2}} i(t)=\frac{\mathrm{d}^{1 / 2}}{\mathrm{~d} t^{1 / 2}} i_{1}(t)+\frac{\mathrm{d}^{1 / 2}}{\mathrm{~d} t^{1 / 2}} i_{2}(t)
$$

After subtracting the contribution of one of the components from the total semidifferentiated signal, the remaining curve can be semiintegrated yielding the original current peak of this component due to the relationship:

$$
\frac{\mathrm{d}^{-1 / 2}}{\mathrm{~d} t^{-1 / 2}}\left(\frac{\mathrm{d}^{1 / 2}}{\mathrm{~d} t^{1 / 2}} i(t)\right)=i(t)
$$

Semidifferentiation and semiintegration can easily be performed with standard algorithms. The problem of removing unwanted peaks from a semiderivative curve and reconstruction of the baseline in these regions must be solved now. Voltammetric peaks which are not very close to each other are sometimes fully separated in the semiderivative domain. This results from the fact that semiderivative peaks are much narrower than voltammetric ones and that the value of the semiderivative drops rapidly to zero at the descending part of the peak, in contrast to the long "diffusional tail" of the original signal (see for example ${ }^{20,21}$ ). This case is shown in Fig. 1A. To remove peak 1, baseline values at points marked $A$ and $B$ must be estimated. Then all datapoints between $A$ and $B$ are replaced by new values calculated under the assumption, that the baseline is linear in this range. Additionally, for the purpose explained later it is 
assumed, that its slope is fairly small. These assumptions are generally valid, if the original voltamperogram is background-corrected.

The situation is more complicated when the semiderivative peaks also overlap (Fig. 1B). The dashed line marks the baseline for peak 1 . The part marked as B is a sum of two contributions: a linear one, corresponding to what was previously called baseline and a non-linear one, being the descending branch of peak 0 in the region where peaks overlap. To be able to remove peak 1 , the curve between $C$ and $A$ must be replaced by the sum of these two components, so it is necessary to estimate both of them separately.

The equation that describes the semiderivative peak of a reversible process is: ${ }^{5,18}$

$$
\begin{aligned}
& \frac{\mathrm{d}^{1 / 2}}{\mathrm{~d} t^{1 / 2}} i=\left(n^{2} F^{2} A v c D^{1 / 2} / 4 R T\right) \cosh ^{-2} \\
& \times {\left[(n F / 2 R T)\left(E-E_{1 / 2}\right)\right] }
\end{aligned}
$$

and it was found, that it can be used for fitting data from irreversible electrode processes, ${ }^{22.23}$ too. For the present purpose, it is also assumed that a function of this type can describe the part of the peak far away from its top, irrespective of the reaction mechanism.

In the considered fragment of the semiderivative voltamperogram, equation (3) can be rewritten in the simplified form

$$
\frac{\mathrm{d}^{1 / 2}}{\mathrm{~d} t^{1 / 2}} i=h \cosh ^{-2}\left[k\left(E-E_{p}\right)\right]
$$

where $h, k$ and $E_{\mathrm{p}}$ denote parameters related to the peak height, to peak width and to the
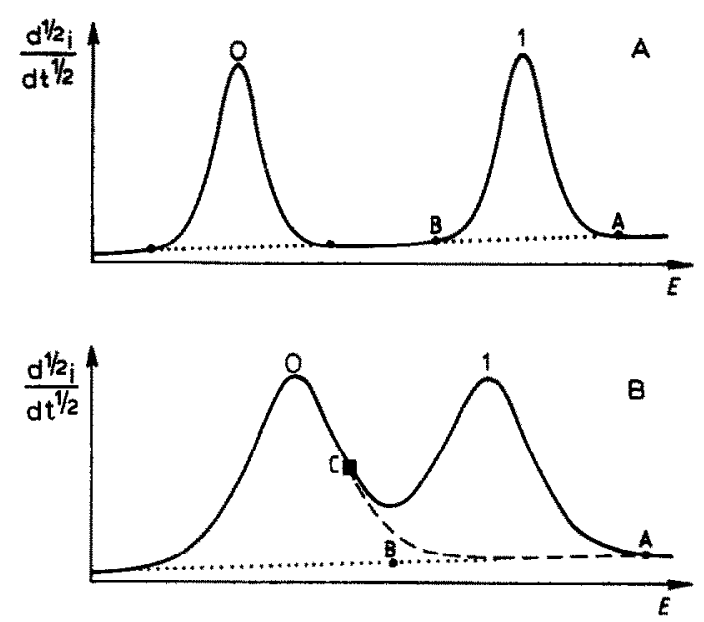

Fig. 1. A-two non-overlapping semiderivative peaks, Small filled circles mark peak borders. B-two overlapping semiderivative peaks. position of peak top, respectively. It must be noted that the above parameters need not necessarily describe the whole peak, but only the relevant fragment.

To characterize the shape of the non-linear component of the baseline, $h, k$, and $E_{\mathrm{p}}$ must be found by curve fitting. Additionally, if datapoints used for fitting are selected from a rather small part of the curve lying, for example, around point $B$ in Fig. 1B, the linear component can be considered constant there. It allows for a reduction in the number of parameters and for the equation for the complete baseline to be written as follows:

$$
y=y_{B}+h_{0} \cosh ^{-2}\left[k_{0}\left(E-E_{\mathrm{p} 0}\right)\right]
$$

where the first term represents the value of the linear component at point $B$ and the second one the descending branch of peak 0 . It should be noted that the slope of the linear component determines the range over which equation (5) is valid.

The overall semi-differentiated signal in the range of selected datapoints can be finally written by adding the function describing the ascending branch of peak 1 to equation (4) resulting in:

$$
\begin{aligned}
y=y_{B}+h_{0} \cosh ^{-2} & {\left[k_{0}\left(E-E_{\mathrm{p} 0}\right)\right] } \\
+ & h_{1} \cosh ^{-2}\left[k_{1}\left(E-E_{\mathrm{pl}}\right)\right]
\end{aligned}
$$

from which the parameters required have to be obtained by curve fitting (indexes indicate peak number). Once $y_{B}$ is found, the linear component of the baseline fragment necessary for the replacement of peak 1 can be obtained by using the value of $y_{A}$ at the point $\mathrm{A}$. Calculation of the $y_{A}$ value at the free end of the overlapping peak can be done in the same manner as calculations at the border points of the non-overlapping peak from Fig. 1A. For these purposes, however, another procedure was used. The method often used of taking the datapoint(s) value(s) at an arbitrary distance from the peak top (far enough to ensure, that the peak contribution is zero) was not employed, because it is subjective and can give wrong results in the case where noise or another peak appears in that region. Instead, an approach was used based on the fitting of datapoints around the peak border point to equation (5) (border point is selected on the basis of peak derivative). In this situation, however, the interpretation of the parameters in equation (5) is slightly different: now $y_{B}$ denotes the wanted value of the baseline at the peak 
border, while the second term is a contribution of the residual of the peak branch. Again, if datapoints are chosen in a narrow range, or if the baseline slope is small, the method yields the baseline value at the border point even if the position of this point was selected not very precisely.

When all necessary baseline parameters are calculated at both peak borders, the unwanted peak can be removed. In case of non-overlapping peaks, it is done just by drawing the straight line between points A and B in Fig. 1A. If the peak overlaps, then the dashed line from Fig. 1B is calculated with the equation

$$
y=y_{0}+s E+h_{0} \cosh ^{-2}\left[k_{0}\left(E-E_{\mathrm{p} 0}\right)\right]
$$

in which $y_{0}$ and $s$ are terms describing the offset and the slope of the linear baseline component (obtained from values of $y_{A}$ and $y_{A}$ and $y_{B}$ ) and the third term is identical to the second term on equation (5), i.e., the descending branch of peak 0 . The procedure proposed can also be used for removing peak 1 instead of peak 0 . In this case, $h_{0}, k_{0}$ and $E_{\mathrm{p} 0}$ in equations (5) and (7) have to be replaced by $h_{1}, k_{1}$ and $E_{p 1}$, respectively.

\section{EXPERIMENTAL}

\section{Reagents}

Solutions $(0.1 \mathrm{M})$ of $\mathrm{In}^{3+}, \mathrm{Tl}^{+}, \mathrm{Co}^{2+}$ and $\mathrm{Zn}^{2+}$ were prepared from indium nitrate, thallium sulphate, cobalt perchlorate (Merck, p.a.) and zinc sulphate (Fluka, p.a.). Potassium chloride $(1 M)$ (Suprapur, Merck) was used as the supporting electrolyte in all experiments. All substances were dissolved in demineralized water filtered through a Millipore Q-II installation. Oxygen was expelled with specially pure (polarographic grade) nitrogen (Hoekloos).

\section{Equipment}

Experiments were carried out with the Autolab-100 fully computer-controlled electrochemical system (Eco Chemie, Utrecht, The Netherlands) connected to a Metrohm 663 VA static mercury drop electrode. $\mathrm{An} \mathrm{Ag} / \mathrm{AgCl}$ electrode ( $3 M$ potassium chloride) was used as the reference electrode. In all experiments. All calculations were done with an Olivetti M24 personal computer (IBM PC/XT compatible).

\section{Recording procedure}

Voltamperograms were recorded with $50 \mathrm{ml}$ of supporting electrolyte. The solution was deaerated for 15-20 min before the experiments.
Two series of measurements for each pair of $A$ and $B$ compounds were done. In the first series, a blank voltamperogram, a voltamperogram of $A$ and a voltamperogram of $A+B$ were recorded by subsequently adding solutions of the respective ions into the cell. The peak of compound $A$, obtained via processing of the $A+B$ voltamperogram were compared to the original voltamperogram of $A$. In the second series, the order of additions was reversed: voltamperograms of a blank, of $B$ and of $B+A$ were recorded. The peak of $B$ separated from the voltamperogram of $B+A$ was compared to the peak of $B$ alone. No correction for the volume change was made, because the total volume change did not exceed $0.2 \%$.

All voltamperograms were stored on disk before further calculations.

\section{Procedure}

The whole procedure for peak separation was implemented in the form of a menu-driven program written in $\mathrm{C}$ (Microsoft-C version). In the development of the program, standard assembler routines from the Autolab-100 analyzer package were used for experimental set-up control.

General scheme. Depending on the kind of studies, voltamperograms were recorded or generated (see below). After completing this step, semidifferentiation was carried out, and baselines of all semiderivative peaks were calculated by curve fitting. All undesired peaks were then removed and replaced by their baselines. The cycle was completed by semiintegration of the processed semiderivative curves yielding the voltamperogram that contains only the peaks(s) of interest.

Data acquisition. Voltamperograms were recorded by varying the potential according to a staircase function and measuring the current in the last $20 \%$ of each step (but no longer than $20 \mathrm{msec}$ ). To reduce the noise, voltamperograms were digitally filtered (Savitzky-Golay procedure ${ }^{24}$ ) or several voltamperograms recorded at subsequent mercury drops were averaged. In the standard procedure, background voltamperograms were always subtracted.

Data generation for simulation purposes. Voltamperograms of reversible systems were generated by use of equation (3). First, the semiderivative peaks for the cathodic and the anodic part were generated, then the whole curve was semiintegrated giving the artificial reversible voltamperogram. Different peak com- 
binations were obtained by changing heights and potentials. The program was capable of generating them for different temperature values as well.

Semidifferentiation/semiintegration. A Grünwald definition of these operations (discrete differintegration $)^{25}$ was used in a modified firstorder algorithm (G0 according to Oldham ${ }^{26}$ ). This algorithm possesses very attractive features: it is fast, simple and can be used for both semidifferentiation and semiintegration (details can be found in the Appendix). There was no significant change in results when the higher order (Gl) algorithm was used. In the implementation presented here, the operation on a curve containing 400 data points took approx. $2.8 \mathrm{sec}$ (IBM PC/XT $8 \mathrm{MHz}$ with coprocessor).

Detection of peak overlapping. The program recognized overlapping and non-overlapping peaks automatically. Test for peak overlapping was based on the check, whether two ranges of datapoints for fitting, as calculated for nonoverlapping peaks (see below), overlap or not.

Fitting of non-overlapping peak border zones. This procedure was used at the free (non-overlapping) ends of peaks. Datapoints for fitting to equation (5) were selected around the peak border within a distance equal to $1 / 6$ of the distance between peak border and peak top. The border of a peak, used in datapoints range selection, was defined as a point, in which the derivative is equal to $0.1 \%$ of its maximal value in the corresponding inflection point. The derivative calculations were carried out with Savitzky-Golay's "smooth derivative" method; ${ }^{24}$ this enables typical noise enhancement caused by differentiation to be overridden. This definition of peak border has several advantages. It is independent of the presence of a baseline with a constant value; due to the very low value of the derivative (practically equal to zero in most situations), if peaks overlap, the border point is placed in the minimum at the curve between them. In case of real voltamperograms containing noise, the border point is selected at the place where noise becomes comparable to the signal. The situation, when the method doesn't give good results is a combination of a very low noise level and a baseline with a large slope.

As initial guesses of the parameters to be fitted, the peak height measured from the curve was used for $h_{0}, 0.1 \%$ of this value as $y_{B}, E_{\mathrm{p} 0}$ was set to the value of the peak potential read from the curve and $k_{0}$ was calculated from the datapoint with highest absolute value, using equation (5).

Fitting of data in the overlap zone. All datapoints constituting the middle $1 / 3$ of the range between peak tops (minimum of 15 points) were fitted to equation (6). This choice was a compromise between requirements of the curve fitting (large number of points spread over the range) and assumptions, under which equation (6) was derived (small range covered by datapoints). Five parameters in this equation $\left(y_{B}, h_{0}, k_{0}, h_{1}\right.$ and $\left.k_{1}\right)$ were fitted, while the other two $\left(E_{\mathrm{p} 0}\right.$ and $E_{\mathrm{pl}}$ ) were directly estimated from the curve by using simple correction because their changes due to overlapping are relatively small. In the first generation of initial guesses, peak potentials (as read from the curve) were used for $E_{\mathrm{p} 0}$ and $E_{\mathrm{pl}}$, for $h_{0}$ and $h_{1}$-directly measured peak heights, $y_{B}$ was set to $0.1 \%$ of the highest value of $h_{0}$ or $h_{1}$ and $k_{0}$ and $k_{1}$ were calculated from the last datapoints at borders of the fitting range assuming, that curve there follows a pure $\cosh ^{-2}$ function. In the next step, the appropriate corrections for the positions of peak tops, due to overlapping, were calculated with guesses from the first generation. The second generation was then created consisting of corrected $E_{\mathrm{p} 0}$ and $E_{\mathrm{pl}}$ values and new $k_{0}$ and $k_{1}$ values re-evaluated with new peak potentials. Finally, $y_{B}, h_{0}, k_{0}, h_{1}$ and $k_{1}$ were used in the curve-fitting procedure for refinement, while $E_{\mathrm{p} 0}$ and $E_{\mathrm{pl}}$ were not corrected further.

The procedure used for curve-fitting was a least-squares one according to Wentworth. ${ }^{27}$ Fitting was performed until corrections to $h_{0}$, $h_{1}, k_{0}$ and $k_{1}$ were smaller than $0.1 \%$.

Removal of undesired peaks. After calculating all the necessary parameters, the baseline was drawn as a straight line, or, baseline points were calculated by using equation (7), depending on whether the removed peak overlaps or not. This procedure covered all points, for which the difference between the original and the new value exceeded $3 \%$.

\section{RESULTS AND DISCUSSION}

The method presented can be applied to the separation of voltammetric peaks in two situations. The first one occurs when both overlapping peaks are present in the same branch, either cathodic or anodic, and the second one occurs when they lie in different branches of the voltamperogram. This latter application deals with the separation of cathodic and anodic 
peaks. It is well known, that in studies of reduction of substances, the anodic peak appears at the "tail" of the cathodic one and consequently the measured anodic current is a sum of the cathodic and anodic contributions (this can easily be seen when the voltamperogram is plotted in $i-t$ coordinates instead of $i-E$ ). From the point of view of peak separation, this situation is similar to the first case, where the latter cathodic peak rises from the "tail" of the previous one. Application of the presented method allows the anodic peak current (an important parameter in mechanistic investigations) to be read directly from the processed voltamperogram.

In the practical part both applications were checked with generated voltammetric curves. To check if the shape of the remaining peak is not influenced by the procedure, four peak parameters were tested: peak potential, peak current and potentials of inflection points. Inflection points were chosen for characterization, because in contrast to the half-peak potentials, their positions are not influenced by a constant or linearly varying background. In order to check the method in reality, two experiments with real electrochemical systems were also performed.

The developed program worked relatively fast: a separation of the voltamperogram of two systems took typically $20-80 \mathrm{sec}$, depending on the noise (which slowed down curvefitting) and the total number of points (increasing differintegration time). In comparison to the method described by Perone et al., ${ }^{3}$ a high number of decisions is done by the program, so no special skill, experience or extensive knowledge about the algorithm principles is demanded from the operator.

\section{Calculations with generated data}

For artificially generated cyclic voltamperograms two series of tests were carried out. In the first, a pair of peaks (cathodic and anodic), simulated for a reversible electrode reaction was separated giving the anodic peak. Because in the process of semidifferentiation only points preceding the investigated one are included in calculations of the semiderivative value, removal of the anodic peak does not influence the cathodic one. The error in the peak current $\left(i_{\mathrm{pa}}\right)$ in separated voltamperograms can be expected to depend on the difference between the peak potential and the potential of the turning point $\left(E_{\mathrm{pc}}-E_{\mathrm{turn}}\right)$.
An example of this separation is shown in Fig. 2. Errors in the anodic peak current, $i_{\text {pe }}$, and the anodic peak potential, $E_{\mathrm{pa}}$, as a function of the difference between cathodic peak potential $\left(E_{\mathrm{pc}}\right)$, and the potential turning point $\left(E_{\text {turn }}\right)$ are collected in Table 1 . It can be seen, that for $E_{\mathrm{pc}}-E_{\text {turn }}>\sim 75 \mathrm{mV}$, the error in peak current was less than $1 \%$, but for very small distances it grew rapidly.

As far as the potential of the anodic peak is concerned, its shift was less than $1 \mathrm{mV}$ if the distance, $E_{\mathrm{pc}}-E_{\mathrm{turn}}$, was $80 \mathrm{mV}$ or more (data points were generated with an interval of 0.98 $\mathrm{mV})$. It is not clear, why two values of errors in $E_{\mathrm{pa}}$, for $E_{\mathrm{pc}}-E_{\mathrm{turn}}$ in range of $200-300 \mathrm{mV}$, deviate from the general trend.

The second test with generated data was carried out in order to find a separation limit for peaks occurring in one branch of the voltamperogram and to study errors in their parameters obtained with the proposed procedure. For this purpose, artificial voltamperograms of two reversible systems were first added up and then separated (heights of cathodic peaks were equal). The parameters obtained were compared with their initial values (an example of the results is presented in Fig. 3, where the voltamperogram containing 2 anodic and 2 cathodic peaks is drawn together with the results obtained-two separated voltamperograms of each system). All results of this test (errors in $i_{p}$, $E_{\mathrm{p}}$ and potentials of inflection points, $E_{\mathrm{asc}}^{\mathrm{inh}}$ and $\left.E_{\text {desc }}^{\text {infl }}\right)$ for all four peaks are collected in Tables $2-5$. It can be seen, that errors are small and have a random character with increasing absolute values for smaller peak distances. Single values deviating from the general trend are due to the discontinuities of the peak function at points where replacement stops. If such discontinuity appears very close to, for example, the inflection point, the obtained respective parameter will be influenced more strongly. The limit of separation, $110 \mathrm{mV}$ for a one-electron process, was determined by the convergence of the curve-fitting. Below this value, real potentials of semiderivative peaks differ significantly from values estimated from the overlapped curve, and fitting doesn't converge. This separation limit appears to be smaller than the one presented in a comparable method. ${ }^{3}$

\section{Experimental data}

The method was checked with real electrochemical systems as well. In two series, a couple of reversible + reversible systems and a couple 


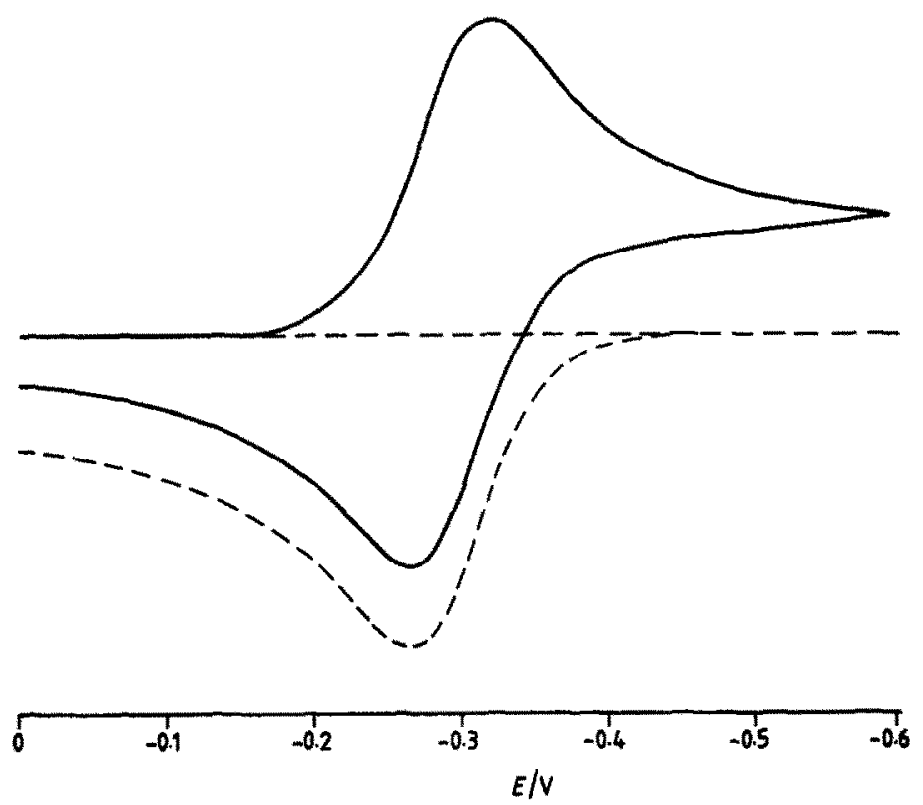

Fig. 2. Generated voltamperogram of one-electron reversible system. Peak potential $E_{\mathrm{pc}}=-0.329 \mathrm{~V}$, potential of turning point $E_{\text {turn }}=-0.6 \mathrm{~V}$, scan rate $0.1 \mathrm{~V} / \mathrm{sec}$, potential step $4 \mathrm{mV}$, temperature $298 \mathrm{~K}$ (solid line) and result of removing the cathodic peak (dashed line).

of non-reversible + non-reversible systems were separated. As an example of the first case, a solution of $\mathrm{In}^{3+}+\mathrm{Tl}^{+}$ions $\left(\Delta E^{0^{\prime}}\right.$ approx. 115 $\mathrm{mV}$ ) was prepared with concentrations of $2 \times 10^{-5}$ and $6 \times 10^{-5} \mathrm{M}$, respectively (voltammetric peaks with approximately equal heights). Voltamperograms were recorded with a scan rate of $0.66 \mathrm{~V} / \mathrm{sec}$ with a potential step of 1.6 $\mathrm{mV}$. Figure 4 presents one of these voltamperograms and two curves obtained as a result of the processing. From the procedure outlined above it could be expected that no baseline remains. However, for the $\operatorname{In}^{3+}$ peak a small potential-dependent baseline is observed, which

Table 1. Errors in the anodic peak potential $\left(E_{\mathrm{pg}}\right)$ and the current $\left(i_{\mathrm{p} \theta}\right)$ as a function of the difference between cathodic peak potential $\left(E_{\mathrm{pc}}\right)$ and turning point potential $\left(E_{\text {turn }}\right)$ for the generated reversible voltamperogram of one system (absolute values). Conditions: $n=1, D_{\text {ox }}=D_{\text {red }}, T=298 \mathrm{~K}$, potential

\begin{tabular}{ccc}
\multicolumn{3}{c}{ step $0.98 \mathrm{mV}$} \\
\hline$E_{\mathrm{pc}}-E_{\text {turn }}, V$ & $\Delta i_{\mathrm{p}} / i_{\mathrm{p}}, \%$ & $\Delta E_{\mathrm{p}}, m V$ \\
\hline 0.041 & 3.69 & 4.5 \\
0.051 & 2.52 & 4.3 \\
0.071 & 1.11 & 3.3 \\
0.096 & 0.4 & 2.6 \\
0.121 & 0.17 & 1.6 \\
0.171 & 0.08 & 0.4 \\
0.221 & 0.04 & 1.7 \\
0.271 & 0.05 & 1.3 \\
0.321 & 0.02 & 0.2 \\
0.421 & 0.09 & 0.3 \\
\hline
\end{tabular}

can be attributed to the small number of points. This number is determined by the lowest potential step height that can be realized with the 12-bits $\mathrm{D} / \mathrm{A}$ converter of the measuring system.

In the second experiment, a mixture of $\mathrm{Zn}^{2+}$ and $\mathrm{Co}^{2+}$ ions (both concentrations $10^{-4} M$ ) in $1 M$ potassium chloride was examined with a scan rate of $1.0 \mathrm{~V}$ and a potential step of $3.6 \mathrm{mV}$. Results of the experiments and experimental conditions are collected in Tables 6 and 7.

In both cases, only cathodic peaks are reported, because with the experimental set-up (relatively small mercury drop) and the potential sweep rate limited by keeping potential step height small, a strong accumulation effect for $\mathrm{In}^{3+}+\mathrm{Tl}^{+}$couple occurred, significantly changing the shape of anodic peaks. This change was large enough to set the convergence limit of curve-fitting above $115 \mathrm{mV}$. In case of the reduction of $\mathrm{Co}^{2+}$ ions, no anodic peak was observed.

All voltamperograms had to be smoothed before processing, because in case of a high $S / N$ ratio, the determination of the peaks borders as described and the curve fitting do not give good results. However, in the authors' experience problem of high noise was encountered very seldom and it was always possible to overcome it by the averaging of several voltamperograms. It should be noted that background subtraction is very important for good performance of the 

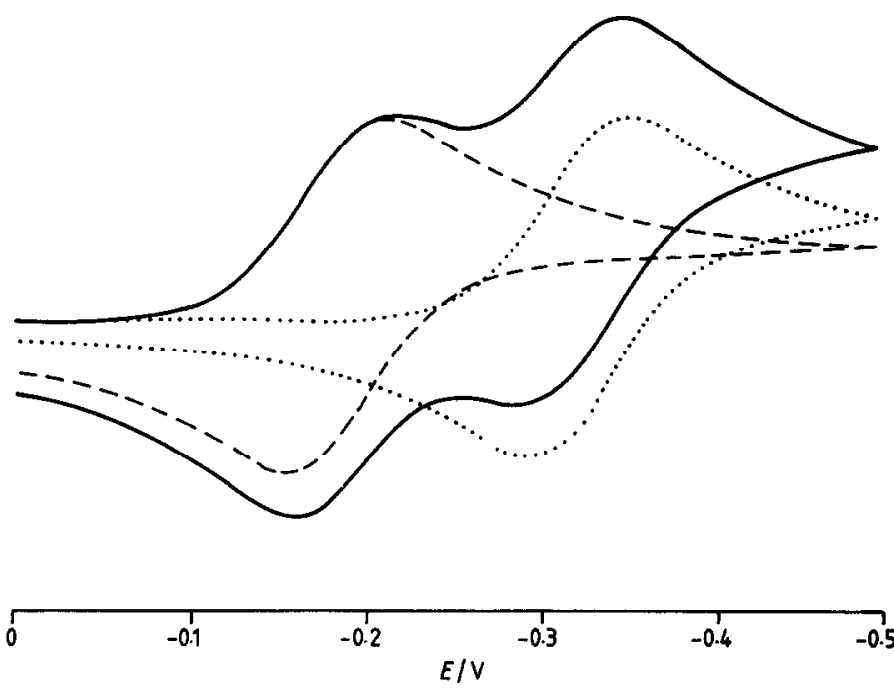

Fig. 3. Solid line: generated voltamperogram of the mixture of two one-electron reversible systems: $E_{\mathrm{pr} 1}=-0.219 \mathrm{~V}, E_{\mathrm{pc} 2}=-0.359 \mathrm{~V}, E_{\mathrm{turn}}=-0.5 \mathrm{~V}$, scan rate $0.1 \mathrm{~V} / \mathrm{sec}$, potential step $2 \mathrm{mV}$; dashed line: result of removing of the contribution of the second system; dotted line: result of removing of the contribution of the first system.

routine because semidifferentiation changes a constant or a linear background of the voltamperograms into baselines of complicated shapes $^{21}$ in the semiderivative domain and assumptions about a linear form of the background become invalid.

Table 2. Errors in peak potentials obtained as a result of separation of single systems from the generated voltamperogram of two reversible systems in dependence on formal potentials $\left(E^{0^{\prime}}\right)$ difference (absolute values). Conditions: equal concentrations, $n_{1}=n_{2}=1$, all diffusion coefficients equal, $T=298 \mathrm{~K}$, potential step $0.98 \mathrm{mV}$. $\mathrm{Cl}$ and $\mathrm{Al}$ refer to peaks of system 1 (easier reducible), $\mathrm{C} 2$ and $\mathrm{A} 2$ refer to system 2

\begin{tabular}{ccccc}
\hline$\Delta E^{0}, V$ & $\Delta E_{\mathrm{pcl}}, m V$ & $\Delta E_{\mathrm{pal}}, m V$ & $\Delta E_{\mathrm{pc} 2}, m V$ & $\Delta E_{\mathrm{pa} 2}, m V$ \\
\hline 0.110 & 0.5 & 0.2 & 1.2 & 0.3 \\
0.115 & 0.4 & 0.4 & 0.8 & 0.9 \\
0.120 & 0.0 & 0.3 & 0.1 & 0.1 \\
0.125 & 0.6 & 0.5 & 0.2 & 0.8 \\
0.130 & 0.6 & 0.3 & 0.7 & 0.3 \\
0.140 & 0.5 & 3.0 & 0.2 & 1.1 \\
0.150 & 2.1 & 0.4 & 0.4 & 2.0 \\
0.160 & 1.5 & 0.4 & 0.4 & 2.0 \\
0.170 & 0.4 & 0.4 & 0.4 & 1.6 \\
\hline
\end{tabular}

Table 3. Errors in peak currents obtained as a result of separation of single systems from the generated voltamperogram of two reversible systems in dependence on formal potentials $\left(E^{\mathbf{\alpha}}\right)$ difference (absolute values). Conditions: equal concentrations, $n_{1}=n_{2}=1$, all diffusion coefficients equal, $T=298 \mathrm{~K}$, potential step $0.98 \mathrm{mV}$. $\mathrm{Cl}$ and $\mathrm{Al}$ refer to peaks of system 1 (easier reducible), $\mathrm{C} 2$ and $\mathrm{A} 2$ refer to system 2

\begin{tabular}{ccccc}
\hline$\Delta E^{0}, V$ & $\Delta i_{\mathrm{pc1}} / i_{\mathrm{pc1}}, \%$ & $\Delta i_{\mathrm{pal}} / i_{\mathrm{pa}}, \%$ & $\Delta i_{\mathrm{pc} 2} / i_{\mathrm{pc} 2}, \%$ & $\Delta i_{\mathrm{pal}} / i_{\mathrm{pal}}, \%$ \\
\hline 0.110 & 0.59 & 1.39 & 0.78 & 1.09 \\
0.115 & 1.23 & 3.19 & 3.17 & 1.55 \\
0.120 & 0.57 & 1.13 & 1.44 & 0.17 \\
0.125 & 1.10 & 1.51 & 1.67 & 0.43 \\
0.130 & 0.5 & 0.82 & 0.11 & 0.28 \\
0.140 & 0.24 & 1.84 & 0.56 & 0.47 \\
0.150 & 0.26 & 0.61 & 0.81 & 0.81 \\
0.160 & 0.56 & 0.57 & 0.74 & 1.23 \\
0.170 & 0.11 & 0.56 & 0.76 & 0.70 \\
\hline
\end{tabular}


Table 4. Errors in potentials of inflection points on ascending branches of peaks obtained as a result of separation of single systems from the generated voltamperogram of two reversible systems in dependence on formal potentials $\left(E^{0}\right)$ difference (absolute values). Conditions: equal concentrations, $n_{1}=n_{2}=1$, all diffusion coefficients equal, $\mathrm{T}=298 \mathrm{~K}$, potential step $0.98 \mathrm{mV} . \mathrm{Cl}$ and $\mathrm{Al}$ refer to peaks of system 1 (easier reducible), $C 2$ and $A 2$ refer to system 2

\begin{tabular}{|c|c|c|c|c|}
\hline$\Delta E^{0^{\prime}}, V$ & $\Delta E_{\mathrm{pcl}}^{\mathrm{nnff,asc}}, m V$ & $\Delta E_{\mathrm{pal}}^{\mathrm{nff,asc}}, m V$ & $\Delta E_{\mathrm{pc} 2}^{\mathrm{nf}, \mathrm{asc}}, m V$ & $\Delta E_{\mathrm{paz}}^{\mathrm{nnf}, \mathrm{asc}}, m V$ \\
\hline 0.110 & 3.5 & 0.2 & 0.0 & 0.2 \\
\hline 0.115 & 1.4 & 0.1 & 0.3 & 1.4 \\
\hline 0.120 & 0.1 & 0.9 & 0.8 & 0.3 \\
\hline 0.125 & 0.7 & 0.5 & 0.5 & 0.8 \\
\hline 0.130 & 0.4 & 0.9 & 0.4 & 0.4 \\
\hline 0.140 & 0.9 & 0.3 & 3.2 & 0.0 \\
\hline 0.150 & 0.3 & 0.5 & 0.6 & 0.7 \\
\hline 0.160 & 0.5 & 3.8 & 4.4 & 0.4 \\
\hline 0.170 & 0.7 & 0.0 & 0.1 & 0.3 \\
\hline
\end{tabular}

Table 5. Errors in potentials of inflection points on descending branches of peaks obtained as a result of separation of single systems from the generated voltamperogram of two reversible systems in dependent on formal potentials $\left(E^{0}\right)$ difference (absolute values). Conditions: equal concentrations, $n_{1}=n_{2}=1$, all diffusion coefficients equal, $\mathrm{T}=298 \mathrm{~K}$, potential step $0.98 \mathrm{mV}$. Cl and $\mathrm{Al}$ refer to peaks of system 1 (easier reducible), $C 2$ and $A 2$ refer to system 2

\begin{tabular}{|c|c|c|c|c|}
\hline$\Delta E^{0^{\prime}}, V$ & $\Delta E_{\mathrm{pcl}}^{\mathrm{tnf}, \mathrm{desc}}, m V$ & $\Delta E_{\mathrm{pa} !}^{\mathrm{nff}, \mathrm{desc}}, m V$ & $\Delta E_{\mathrm{pc} 2}^{\mathrm{nn} \text {,desc }}, m V$ & $\Delta E_{\mathrm{pa2} 2}^{\mathrm{uff} \text {,desc }}, m V$ \\
\hline 0.110 & 0.2 & 0.4 & 0.0 & 1.1 \\
\hline 0.115 & 0.5 & 0.7 & 0.7 & 0.6 \\
\hline 0.120 & 0.4 & 0.5 & 0.3 & 0.5 \\
\hline 0.125 & 0.6 & 0.5 & 0.3 & 0.4 \\
\hline 0.130 & 0.4 & 0.4 & 0.1 & 0.4 \\
\hline 0.140 & 0.5 & 0.3 & 0.2 & 0.6 \\
\hline 0.150 & 0.7 & 0.7 & 0.3 & 0.8 \\
\hline 0.160 & 0.4 & 0.7 & 0.3 & 0.3 \\
\hline 0.170 & 2.1 & 1.1 & 0.3 & 0.9 \\
\hline
\end{tabular}

\section{CONCLUSIONS}

The method presented here can be applied to the resolution of overlapping LSV or CV peaks originating from both reversible and non-reversible systems with reliable results. When used for the separation of peaks in one branch (cathodic or anodic), a resolution limit of $110 \mathrm{mV}$ (reversible case, one electron transfers) could be obtained.

When resolving peaks lying in different branches of the cyclic voltammetric curve, the anodic and cathodic peak of the investigated system, free from a cathodic or anodic peak influence, respectively, can be obtained. Precision of the calculated peak parameters depends in this case on the distance between peak top and the turning point of the voltamperogram.

Accumulation in the mercury drop influences the shape of the semiderivative peaks in the anodic segment and increases the minimal distance between the two peaks that can be resolved. When two voltammetric peaks strongly overlap and the accumulation effect occurs, the anodic part of the voltamperogram sometimes cannot be separated even though the cathodic part can.

The algorithm needs hardly any decisions by the operator, which is an attractive feature when

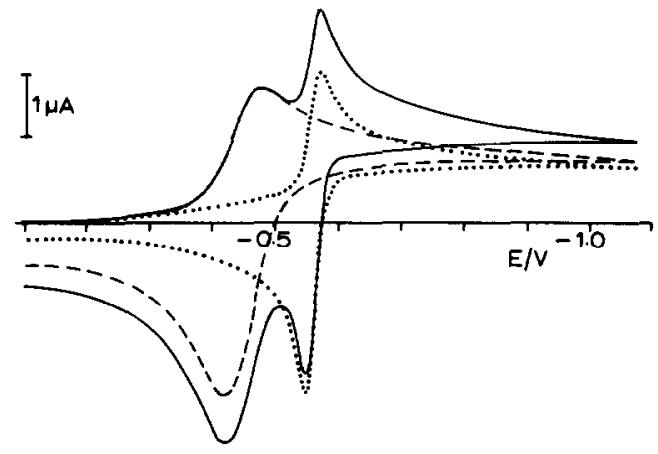

Fig. 4. Experimental cyclic voltamperogram of the $\mathrm{Tl}^{+}+\mathrm{In}^{3+}$ mixture (experimental conditions as in Table 6) (solid line) and the individual contributions calculated by the procedure described: dashed line: $\mathrm{Tl}^{+}$ion contribution, dotted line- $\mathrm{In}^{3+}$ contribution. 
Table 6. Errors in parameters of the cathodic peak obtained by the separation of peak of interest from a voltamperogram of two reversible systems-experimental data. Experimental conditions: solution $\mathrm{In}^{3+}+\mathrm{Tl}^{+}\left(2 \times 10^{-5} M+6 \times 10^{-5} M\right.$ in $\left.1 M \mathrm{KCl}, \Delta E^{0} 115 \mathrm{mV}\right)$, scan rate: $0.66 \mathrm{~V} / \mathrm{sec}$, potential step: $1.6 \mathrm{mV}$

\begin{tabular}{lcccc}
\hline System & $\Delta i_{\mathrm{p}} / i_{\mathrm{p}}, \%$ & $\Delta E_{\mathrm{p}}, m V$ & $\Delta E_{\mathrm{ase}}^{\mathrm{inf}}, m V$ & $\Delta E_{\text {desc }}^{\text {inf }}, m V$ \\
\hline $\mathrm{T}^{+} / \mathrm{T} 1$ & -0.31 & -2.3 & -0.7 & -2.8 \\
$\mathrm{In}^{3+} / \mathrm{In}$ & -0.31 & -1.8 & -0.4 & -1.5 \\
\hline
\end{tabular}

Table 7. Errors in parameters of the cathodic peak obtained by separation of the peak of interest from a voltamperogram of two irreversible systems-experimental data. Experimental conditions: solution $\mathrm{Zn}^{2+}+$ $\mathrm{Co}^{2+}\left(10^{-4} M+10^{-4} M\right.$ in $\left.1 M \mathrm{KCl}, \Delta E_{\mathrm{p}} 440 \mathrm{mV}\right)$, scan rate: $1.0 \mathrm{~V} / \mathrm{sec}$, potential step: $3.6 \mathrm{mV}$

\begin{tabular}{lcccc}
\hline System & $\Delta i_{\mathrm{p}} / i_{\mathrm{p}}, \%$ & $\Delta E_{\mathrm{p}}, m V$ & $\Delta E_{\mathrm{asc}}^{\mathrm{nnf}}, m V$ & $\Delta E_{\text {desc }}^{\text {nnf }}, m V$ \\
\hline $\mathrm{Zn}^{2+} / \mathrm{Zn}$ & +1.6 & -1 & -5 & +1.2 \\
$\mathrm{Co}^{2+} / \mathrm{Co}$ & +5.8 & -2 & -3 & -9 \\
\hline
\end{tabular}

the automatic processing of voltamperograms is concerned. This implies that the program can be used by personnel having little experience in using electrochemical methods.

\section{APPENDIX}

The Grünwald definition of discrete differintegration is:

$$
\frac{\mathrm{d}^{u} f\left(t_{j}\right)}{\mathrm{d} t^{u}}=\lim _{N->\infty}\left[\frac{\left(N / t^{\prime}\right)^{u}}{\Gamma(-u)} \sum_{j=1}^{N} \frac{\Gamma(N-j-u)}{\Gamma(N-j+\mathrm{I})} f\left(t_{j}\right)\right]
$$

where $f(t)$ is a value of the function to be differintegrated at time $t_{f}, N$ is the number of evenly spaced points preceding the point $t, t^{\prime}$ is the time interval between points, $u$ is the instance of differintegration (1/2 for semidifferentiation and $-1 / 2$ for semiintegration). This equation can be rewritten in the convolutional form

$$
\begin{aligned}
\frac{\mathrm{d}^{u} f\left(t_{j}\right)}{\mathrm{d} t^{u}} & =\lim _{N->\infty}\left[\left(N / t^{\prime}\right)^{u} \sum_{j=1}^{N} \frac{\Gamma(N-j-u)}{\Gamma(-u) \Gamma(N-j+1)} f\left(t_{j}\right)\right] \\
& =\lim _{N \rightarrow>\infty}\left[\left(N / t^{\prime}\right)^{u} \sum_{j=1}^{N} w_{j} f\left(t_{j}\right)\right]
\end{aligned}
$$

where $w$, are values of a weighting function depending only on $u$ and $N$. This reformulation gives an approach equivalent to the $\mathrm{G} 0$ algorithm described by Oldham. ${ }^{26}$

Inspection of the last $w_{3}$ values gives:

$$
\begin{aligned}
W_{N} & =\frac{\Gamma(N-N-u)}{\Gamma(-u) \Gamma(N-N+1)}=1 \\
w_{N-k} & =\frac{\Gamma(N-N+k-u)}{\Gamma(-u) \Gamma(N-N+k+1)}=\frac{\Gamma(k-u)}{\Gamma(-u) \Gamma(k+1)} \\
W_{N-k-1} & =\frac{\Gamma(N-N+k+1-u)}{\Gamma(-u) \Gamma(N-N+k+2)} \\
& =\frac{(k-u) \Gamma(k-u)}{(k+1) \Gamma(-u) \Gamma(k+1)}=\frac{k-u}{k+1} W_{N-k}
\end{aligned}
$$

and leads to the general recursive formula

$$
W_{j}=\frac{k-u}{k+1} W_{j+1}, \quad W_{N}=1
$$

where $k$ is an index counting from the end, $k=N-j-1$.
This definition makes the weighting function independent of the value of $N$. It can be found, that for differintegration of a set of $N$ points it is enough to calculate the set of $N$ weights only once at the beginning and then differintegration becomes a summation of weighted point values. This approach allows one to make a very compact and simple algorithm for differintegration, in which, after calculation of weights, only $n$ multiplications and $n-1$ additions (plus one multiplication by a normalizing factor) are to be done in order to calculate the differintegral value in $j$-th point.

\section{GLOSSARY}

$A \quad$ electrode surface area

c concentration

$D$ diffusion coefficient

$E \quad$ actual electrode potential

$E_{1 / 2} \quad$ potential of polarographic halfwave of the compound

$E_{\mathrm{p} 0}, E_{\mathrm{p} 1}$ parameters related to potential at which semiderivative peak \#0 and \#1 appear

$F \quad$ Faraday constant

$h \quad$ semiderivative peak height

$h_{0}, h_{1} \quad$ parameters related to height of respectively semiderivative peak \#0 and \#1

$i, i(t) \quad$ current

$k$ parameter describing width of the semiderivative peak

$k_{0}, k_{1} \quad$ parameters related to width of respectively semiderivative peak \# 0 and \#1 number of electrons involved in electrode process actual number of the point, counting from the lower limit of the semiderivative slope of the baseline in semiderivative domain

absolute temperature 
$t^{\prime} \quad$ time interval between two subsequent points

$u \quad$ instance of differintegration

$v \quad$ scan rate

$w_{j} \quad$ weight for point $j$

$y \quad$ value of semiderivative of the current in respect to time

$y_{A}, y_{B}$ baseline value at semiderivative peak border

$y_{0}$ constant term in linear baseline equation, obtained from combination of baseline values at both peak borders.

\section{REFERENCES}

1. W. F. Gutknecht and S. P. Perone, Anal. Chem. 1970, 42, 906.

2. J. F. Rusling and T. F. Connors, ibid., 1983, 55, 776.

3. S. P. Perone, J. W. Frazer and A. Kray, ibid., 1971, 43, 1485.

4. P. A. Boudreau and S. P. Perone, ibid., 1979, 51, 811.

5. M. Goto and D. Ishii, J. Electroanal. Chem. 1975, 61, 361 .

6. P. Dalrymple-Alford, M. Goto and K. B. Oldham, Anal. Chem. 1977, 49, 1390.
7. K. B. Oldham, ibid., 1972, 44, 196.

8. M. Goto and D. Ishii, J. Electroanal. Chem. 1979, 102, 49.

9. K. B. Oldham and J. Spanier, ibid., 1970, 26, 331.

10. C. P. Andrieux, L. Nadjo and J. M. Saveant, ibid., 1970, 26, 147.

11. J. C. Myland, K. B. Oldham and C. G. Zoski, ibid., 1985, 193, 3.

12. J. C. Imbeaux and J. M. Savéant, ibid., 1973, 44, 169.

13. F. Ammar and J. M. Savéant, ibid., 1973, 47, 215.

14. L. Nadjo, J. M. Savéant and D. Tessier, ibid., 1974, 52 , 403.

15. J. M. Savéant and D. Tessier. ibid., 1975. 61, 251.

16. Idem, ibid., 1975, 65, 57.

17. Idem. ibid., 1977, 77, 225.

18. P. Dalrymple-Alford, M. Goto and K. B. Oldham, ibid., $1977,85,1$.

19. K. B. Oldham, S.I.A.M. J. Math. Anal. 1983, 14, 974.

20. D. E. Smith, Anal. Chem. 1976, 48, 517A.

21. K. B. Oldham and C. G. Zoski, J. Electroanal. Chem. 1983, 145, 265.

22. J. J. Toman and S. D. Brown, Anal. Chem. 1981, 53, 1497.

23. D. M. Caster, J. J. Toman and S. D. Brown, ibid., 1983, 55, 2143.

24. A. Savitzky and M. J. E. Golay, ibid., 1964, 36, 1627.

25. K. B. Oldham and J. Spanier, The Fractional Calculus, Academic Press: New York, London, 1974.

26. K. B. Oldham, J. Electroanal. Chem. 1981, 121, 341.

27. W. E. Wentworth, J. Chem. Ed. 1965, 42, 96. 